\title{
Evaluation of vascular access before kidney transplantation in patients with successful kidney transplantation
}

\author{
Mohsen Mohammad Rahimi ${ }^{1}{ }^{\circledR}$, Mansour Alizadeh $^{1}$, Nima Naghdi-seded $^{2}$, Rohollah Valizadeh $^{3}$, \\ Amir Jamei ${ }^{1}$, Aarefeh Jafarzadeh-Kohneloo ${ }^{4}$, Shokoofeh Dorosti ${ }^{5}$, Saman Farshid ${ }^{* \circledast}$ \\ ${ }^{1}$ Nephrology and Kidney Transplant Research Center, Urmia University of Medical Sciences, Urmia, Iran \\ ${ }^{2}$ Urologist, Tabriz University of Medical Science, Tabriz, Iran \\ ${ }^{3}$ Student Research Committee, Urmia University of Medical Science, Urmia, Iran and PhD Student in Epidemiology, Student Research \\ Committee, Iran University of Medical Science, Tehran, Iran \\ ${ }^{4}$ Department of Epidemiology and Biostatistics, Faculty of Health, Tehran University of Medical Sciences, Tehran, Iran \\ ${ }^{5}$ Urmia University of Medical Sciences, Urmia, Iran
}

\section{A R T I C L E I N F O}

Article Type:

Original

\section{Article History:}

Received: 2 March 2018

Accepted: 14 May 2018

Published online: 19 May 2018

\section{Keywords:}

Kidney transplantation

Fistula

Vascular access

\begin{abstract}
A B S T R A C T
Introduction: The status of embedded fistula before kidney transplant is one of the problems in patients after kidney transplant and without correct management, surely it can lead to severe complications. The vascular access of arteriovenous fistula (AVF) and its possible complications can be found using the registered data in patients who had undergone successful kidney transplant. This information may be useful to prevent possible complications in future. Objectives: This study was aimed to determine the status of vascular access before kidney transplant in patients with successful transplantation in Urmia city, Iran.

Patients and Methods: This study was a historical cohort conducted on 201 patients from March 2009 to March 2012 who had undergone vascular access before kidney transplant. The related information about complications and functions of fistula were extracted from available records.

Results: According to results, the patients were divided into four groups regarding fistula function including 104 patients (51.7\%) with functional fistula after kidney transplant, 49 patients $(24.4 \%)$ with spontaneously closed fistula, 37 patients $(18.4 \%)$ with closed fistula through surgery and 11 patients $(5.5 \%)$ with reduced blood flow of fistula.

Conclusion: Despite the low incidence of heart failure in functional vascular access after successful renal transplantation, these patients should be followed up by cardiologists to avoid complications. Generally, closing vascular accesses after successful transplantation is not necessary.
\end{abstract}

\section{Implication for health policy/practice/research/medical education:}

Despite the low incidence of heart failure in functional vascular access after successful renal transplant, these patients should be followed up by cardiologists to avoid complications. Generally, closing the vascular access after successful transplantation is not necessary.

Please cite this paper as: Mohammad Rahimi M, Alizadeh M, Naghdi-seded N, Valizadeh R, Jamei A, Jafarzadeh-Kohneloo A, et al. Evaluation of vascular access before kidney transplantation in patients with successful kidney transplantation. J Renal Inj Prev. 2019;8(1):50-53. Doi: 10.15171/jrip.2019.10.

\section{Introduction}

The status of embedded fistula before kidney transplant is one of the problems in patients after kidney transplant surgery while it will lead to complications when it cannot be correctly managed. In fact, it is a question for most patients after successful kidney transplant whether fistulas should be retained for probable dialysis. Arteriovenous fistula (AVF) has more hazards, high costs and abundant morbidities in hemodialysis patients (1). The major complications of AVF are bleeding, infection, nerve injury, the lack of early function, thrombosis, stenosis, steel syndrome and aneurysm (2). Physicians are advised 
to keep the fistula in patients with kidney transplant but this advice is sometimes challenging. On the other hand, there is no single strategy to maintain or close fistula $(3,4)$. The closure of the fistula can be considered as an indication of the occurrence of complication, heart failure and increased blood flow of fistula. The closure of the AVF can reduce the volume and mass of left ventricle and it can be detected in the successful kidney transplant surgery in patients with left ventricular dilatation (4). As mentioned, there is no single protocol to treat AVF after kidney transplant and it needs more attention to find a suitable decision. Additionally, in some studies the relationship between the survival of fistula and survival of kidney transplant has been noted more important. In our country, there are a few studies regarding the effect of AVF on left ventricular function of the heart and other organs. Hoverer, there are few studies about the other complications of fistula in kidney transplanted patients and the natural process of fistula. In the Imam Khomeini hospital of Urmia in West Azerbaijan of Iran, approximately 100 kidney transplant surgeries were done annually while the majority of them had vascular access before surgery. The vascular access of AVF and its possible complications can be found using the registered data in patients who had undergone a successful kidney transplant. Additionally, this information can be useful to prevent probable complications.

\section{Objectives}

This study was aimed to determine the status of vascular access before kidney transplantation in patients with successful surgery in Imam Khomeini hospital of Urmia city during 2007-2012.

\section{Patients and Methods}

\section{Patient selection}

This retrospective study was conducted on 201 patients who had undergone vascular access before kidney transplantation from March 2009 to March 2012. The related information about complications and functions of fistula was based on color Doppler ultrasound and clinical examinations which were extracted from records. Spontaneous closure of the fistula is defined as a lack of thrill in touch under clinical examination. Increased and decreased blood flow of fistula were different based on the area of fistula. The patients who had not underwent embedded vascular access in these 3 years (2007-2010), they were not included in the study and it is considered as exclusion criteria. In addition, patients who had not completed records were excluded from the study. Therefore, the natural process and function of fistula during three years after kidney transplant were evaluated.

\section{Ethical issues}

1) The research followed the tenets of the Declaration of Helsinki; 2) written informed consent was obtained, and they were free to leave the study at any time; and 3) the research was approved by the ethical committee of Urmia University of Medical Sciences.

\section{Statistical analysis}

The data were analyzed using SPSS version 18 and descriptive data for continuous variables and qualitative statistics were used as pie, bar charts and tables.

Results

This study included 201 patients who had undergone successful kidney transplant. We evaluated the natural process of AVF after kidney transplant. The mean age of patients was $41.1 \pm 14.54$ years. Of a total 201 patients, 120 patients $(57.9 \%)$ were male and 81 patients $(40.3 \%)$ were female. Regarding area of vascular access, 47 patients (23.4\%), had vascular access in elbow, around 95 patients (47.35) had AVF in the snuff box while 43 patients had AVF (21.4\%) in arteriovenous arm and finally 16 patients had AVF (8\%) in the other places (Table 1 and Figure 1). According to the results, patients were divided into four groups regarding fistula function including 104 patients (51.7\%) with functional fistula after kidney transplant, 49 patients $(24.4 \%)$ with spontaneously closed fistula, 37 patients $(18.4 \%)$ with closed fistula through surgery and 11 patients (5.5\%) with reduced blood flow of fistula (Table 2 and Figure 2).

Of a total 201 patients, only 4 patients (2\%) experienced heart complications (Table 3 ).

In this study, the causes of end-stage kidney failure patients were; hypertension in 86 patients (42.8\%), glomerulonephritis in 47 patients (23.4\%), diabetes in 30 patients (14.9\%) and other causes in 38 patients (18.9\%), respectively (Table 4 and Figure 3 ).

Table 1. Frequency of vascular access placement according to area

\begin{tabular}{lcc}
\hline Area & No. & Percent \\
\hline Elbow & 47 & 23.4 \\
Snuffbox & 95 & 47.3 \\
Brachioaxillary graft & 43 & 21.4 \\
Other & 16 & 8.0 \\
Total & 201 & 100.0 \\
\hline
\end{tabular}

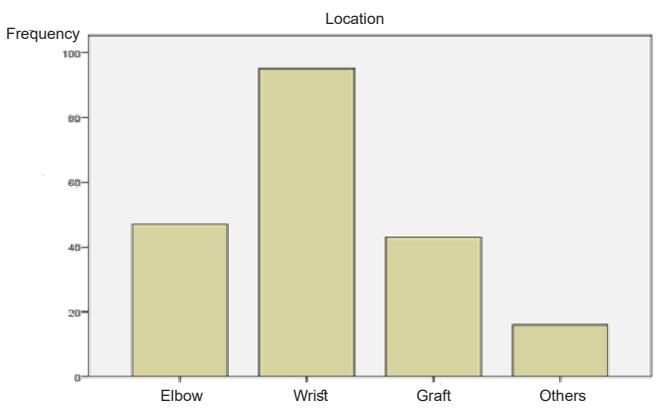

Figure 1. Frequency of embedded vascular access regarding their area in studied patients. 
Table 2. Frequency of natural process of vascular access regarding function after kidney transplant surgery

\begin{tabular}{lll}
\hline Outcome & No. & Percent \\
\hline Normal function & 104 & 51.7 \\
Spontaneous closure & 49 & 24.4 \\
Surgical closure & 37 & 18.4 \\
Decreased blood flow & 11 & 5.5 \\
Total & 201 & 100.0 \\
\hline
\end{tabular}

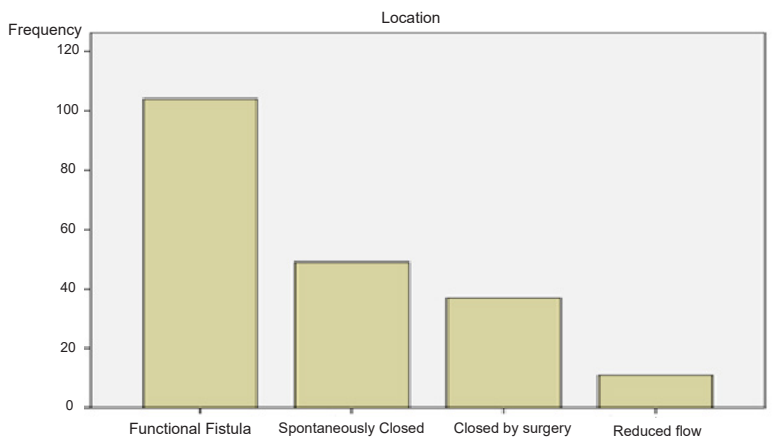

Figure 2. Frequency of natural process of vascular access in studied patients.

Table 3. Frequency of vascular access complications after kidney transplant

\begin{tabular}{lll}
\hline Complication & No. & Percent \\
\hline No & 197 & 98.0 \\
Yes & 4 & 2.0 \\
Total & 201 & 100.0 \\
\hline
\end{tabular}

Table 4. Causes of kidney failure in patients

\begin{tabular}{lll}
\hline Cause of kidney failure & Frequency & Percent \\
\hline Diabetes & 30 & 14.9 \\
Glomerulonephritis & 47 & 23.4 \\
Blood pressure & 86 & 42.8 \\
Others & 38 & 18.9 \\
Total & 201 & 100.0 \\
\hline
\end{tabular}

According to results of 201 patients, 162 (80.6\%) were without complications, 20 patients (51.3\%) experienced edema, 10 patients (25.6\%) experienced infection, 7 patients $(17.9 \%)$ experienced increased blood flow and 2 patients $(5.1 \%)$ experienced severe thrombosis (Table 5 and Figure 4).

\section{Discussion}

In this study, patients were divided into four groups regarding fistula function including 104 patients (51.7\%) with functional fistula after kidney transplant, 49 patients (24.4\%) with spontaneously closed fistula, 37 patients (18.4\%) with closed fistula through surgery and 11 patients (5.5\%) with reduced blood flow of fistula. In the study of Patard et al, conducted on 160 patients with kidney transplant, the natural process of fistulas was evaluated

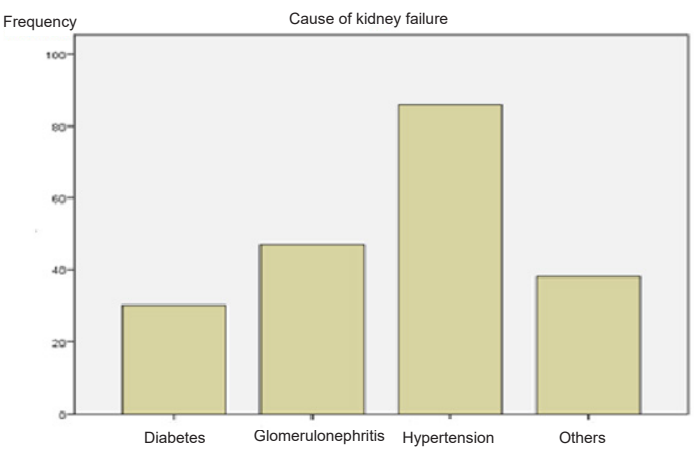

Figure 3. The causes of renal failure in patients.

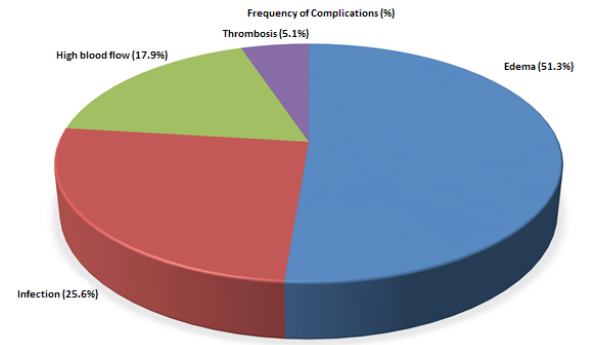

Figure 4. Frequency of complications of vascular access in patients.

Table 5. Frequency of vascular access complications after kidney transplant

\begin{tabular}{lcc}
\hline Complication & No. & Percent \\
\hline Venous edema & $r$. & $\Delta I, r$ \\
Infection & . & $r \Delta / 9$ \\
High flow & $V$ & $I V / 9$ \\
Thrombosis & $r$ & $\Delta / 1$ \\
\hline
\end{tabular}

after surgery and $7.5 \%$ of fistulas closed spontaneously, as well as $61 \%$ of fistulas were functional until the end of the study (5). These results were consistent with our study, given that the rate of spontaneous closure was somewhat more in their study.

Likewise, Manca et al showed that among 365 patients that had good kidney function after kidney transplant, 198 patients (54.2\%) had functional fistula and 167 patients (45.8\%) had no functional fistula, while125 patients (34.2\%) experienced spontaneous closure and 42 patients (11.5\%) had surgical closure (6). These results were consistent with our study while the majority of vascular access were functional after kidney transplantation. In our study, of 201 patients, only 4 patients (2\%) had heart complication caused by fistulas, but there were 6 patients with heart complication caused by fistulas in the study by Vajdič Trampuž et al (7). Additionally, in the studies of Soleimani et al and Sheashaa et al, spontaneous closure of the fistula had no effect on heart performance $(8,9)$.

Despite having high heart index and output, in the study of Sheashaa et al, no significant difference between patients 
with functional fistula and patients with nonfunctional fistula was detected (9). The prevalence and impact of functional fistula on kidney function was negligible according to the mentioned studies and our study. However, more detailed studies are needed to investigate this issue.

In our study, 162 patients ( $80.6 \%$ ) had no complications, 20 patients $(51.3 \%)$ experienced edema, 10 patients $(25.6 \%)$ experienced infection, 7 patients (17.9\%) experienced increasing blood flow and 2 patients (5.1\%) experienced severe thrombosis. In the study conducted by Vajdič Trampuž et al, the venous complications were evaluated on 74 patients who had kidney transplant surgery. The complications were observed in 32 patients (43.2\%) with painful thrombosis (with or without phlebitis), 26 patients (27\%) with aneurysm, 6 patients $(8.1 \%)$ with venous hypertension with arm edema, 6 patients $(8.1 \%)$ with distal hypoperfusion, 6 patients(8.1\%) with increased blood flow and heart failure, one patient (1.3\%) with trauma and 4 patients (4\%) with cosmetic complications (7).

The results of their study were inconsistent with our study because of different complication rates, while, it was low in our study. Similarly, the rate of venous edema was more than our study. Additionally in the study of Vajdič Trampuž et al (7), the rate of venous complications was more than our investigation. The cause of this difference may be due to poor registry system and also lack of appropriate follow-up by the patients.

\section{Conclusion}

Despite the low incidence of heart failure in functional vascular access after successful kidney transplantation, these patients should be followed up by cardiologists to avoid complications. Additionally, to avoid other complications in these patients, it is recommended to be visited regularly.

\section{Limitations of the study}

Generally, the closing of vascular access after successful kidney transplant does not seem necessary, if there is no complication. However, further studies with larger sample size are needed to investigate the effect of functional vascular access complications in the survival of patients with kidney transplant using prospective cohort study.

\section{Authors' contribution}

MMR, MMF and SF conducted the research. NNS, AJK and SD collected the data. NNS and SF analyzed the data. RV prepared the primary draft. NNS, MMR and SF edited the final draft. All authors signed the manuscript.

\section{Conflicts of interest}

The authors declared that there was no conflict of interest in this study.

\section{Ethical considerations}

Ethical issues (including plagiarism, data fabrication, double publication) have been completely observed by the authors.

\section{Funding/Support}

This study is supported by Urmia University of Medical Sciences.

\section{References}

1. Bourquelot P, Levy BI. Narrow elastic disposable tourniquet (Hemaclear(R)) vs. traditional wide pneumatic tourniquet for creation or revision of hemodialysis angioaccesses. J Vasc Access. 2016;17:205-9. doi: 10.5301/jva.5000520.

2. Unger P, Wissing KM, de Pauw L, Neubauer J, van de Borne P. Reduction of left ventricular diameter and mass after surgical arteriovenous fistula closure in renal transplant recipients. Transplantation. 2002;74:73-9.

3. van Duijnhoven EC, Cheriex EC, Tordoir JH, Kooman JP, van Hooff JP. Effect of closure of the arteriovenous fistula on left ventricular dimensions in renal transplant patients. Nephrol Dial Transplant. 2001;16:368-72. doi: 10.1093/ ndt/16.2.368.

4. Russell TE, Kasper GC, Seiwert AJ, Comerota AJ, Lurie F. Cilostazol may improve maturation rates and durability of vascular access for hemodialysis. Vasc Endovascular Surg. 2017;51:120-124. doi: 10.1177/1538574417692464

5. Patard JJ, Bensalah K, Lucas A, Rodriguez A, Manunta A, Rivalan J, et al. Management of vascular access for hemodialysis after successful kidney transplantation. Scand J Urol Nephrol. 2002;36:373-6. doi: $10.1080 / 003655902320783890$

6. Manca O, Pisano GL, Carta P, Manca EM, Piredda GB, Pili $\mathrm{G}$, et al. The management of hemodialysis arteriovenous fistulas in well-functioning renal transplanted patients: many doubts, few certainties. J Vasc Access. 2005; 6:182-6.

7. Vajdič Trampuž B1, Ponikvar R, Kandus A, ButurovićPonikvar J. Hemodialysis arteriovenous fistula-related complications and surgery in kidney graft recipients. Ther Apher Dial. 2013;17:444-7. doi: 10.1111/1744-9987.12094.

8. Soleimani MJ, Shahrokh H, Shadpour P, Shirani M, Arasteh S. Impact of dialysis access fistula on cardiac function after kidney transplantation. Iran J Kidney Dis. 2012;6:198-202. doi: $10.1111 /$ tri.12326

9. Sheashaa H, Hassan N, Osman Y, Sabry A, Sobh M. Effect of spontaneous closure of arteriovenous fistula access on cardiac structure and function in renal transplant patients. Am J Nephrol. 2004;24:432-7. doi:.10.1159/000080187

Copyright ( $) 2019$ The Author(s); Published by Nickan Research Institute. This is an open-access article distributed under the terms of the Creative Commons Attribution License (http://creativecommons.org/licenses/by/4.0), which permits unrestricted use, distribution, and reproduction in any medium, provided the original work is properly cited. 